\title{
Discharge Currents Discrimination Technique Based on Multi-Linear Regression Line and Artificial Neural Networks for Power Transformers Diagnosis
}

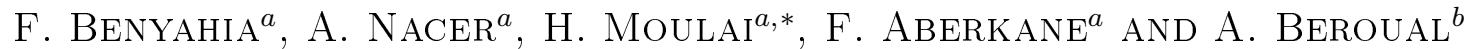 \\ ${ }^{a}$ Laboratory of Electrical and Industrial Systems, FEI, USTHB, BP 32 Bab Ezzouar, Algiers, 16311, Algeria \\ ${ }^{b}$ Ecole Centrale de Lyon, Ampere CNRS UMR 5005, 36 av. Guy de Collongue, 69134 Ecully, France \\ The proposed work will be consecrated to the study of positive pre-breakdown currents triggered in mineral \\ transformer oil under $50 \mathrm{~Hz}$ alternating overvoltage. Since negative currents are recorded in low rates and for higher \\ voltage levels than positive ones, only the latter will be prior taken into consideration. Both streamer propagation \\ and arc discharge current types are identified and are used in the training process of an artificial neural network and \\ the multi-linear regression line of these currents in order to develop a complementary diagnosis tool that can serve \\ as an on-line transformer protection. More successful results than those obtained by other developed techniques \\ are expected.
}

DOI: 10.12693 /APhysPolA.123.250

PACS: $77.22 . \mathrm{Jp}, 52.80 . \mathrm{Wq}$

\section{Introduction}

Power transformers are mineral insulating oil filled, which serves for both cooling and insulating of their different active parts. Most of their failures are due to electrical discharges occurrence and overheating. The physical nature of the discharges must be well identified as a function of their energy in order to take a suitable action to protect this equipment.

Indeed, electrical arcing discharges in liquid dielectrics are generally preceded by streamer generation and propagation through the electrode gap. Their propagation velocity and corresponding current are greater than the streamers ones, and depend mainly on the nature of the liquid and the applied electric field [1]. Furthermore, the transition of a streamer to an arcing discharge obeys a statistical law [2].

The pre-breakdown and breakdown phenomena in liquid dielectrics have been the subject of numerous studies mainly consecrated to the shape and velocity of the streamers as well as to the electric, optic, and acoustic parameters that characterize them. Currents, correlated emitted lights, and acoustic echoes are privileged parameters for sensor inventors that can serve as elements of supervision and decision for the protection of the equipments in which these oils are used.

Several techniques of measurement and diagnosis have been then proposed for power transformer monitoring. Most of them are plainly performed out of service. They sometimes come very tardily, especially when the process of deterioration is already accelerated. For that purpose, new surveillance live techniques have been developed [3-5] as complementary to the classical methods

*corresponding author; e-mail: moulaih@yahoo.fr
[6-8]. They are based on numerical techniques leading to more satisfactory results.

Since positive streamers are initiated earlier than the negative ones [2], this work will be focused on positive streamers occurrence and their probability to lead to arc discharges. An artificial neural network (ANN) will be developed in the purpose of surveillance and protection of power transformers against such discharges succeeding to positive streamers propagation under alternating voltage. This will enable to consider the transformers protection at the ultimate instant against internal discharges of high energy level.

\section{Experimental technique}

The experimental device is constituted by a HV transformer $(220 \mathrm{~V} / 50 \mathrm{kV}, 50 \mathrm{~Hz})$ and a test cell made of Pyrex of $500 \mathrm{ml}$ volume, containing a point-plane electrode system. The plane electrode is a brass circular disc of $40 \mathrm{~mm}$ diameter, covered with an insulating sheet of plexiglas in order to avoid eventual damages to the measuring apparatus. The point electrode is a tungsten type whose radius curvature is $10 \mu \mathrm{m}$. The electrode gap $d$ is varied between 2.0 and $18.5 \mathrm{~mm}$.

\section{Current measurement}

The streamer current is measured through a non-inductive resistance $R_{m}$ of $50 \Omega$ placed between the plane electrode and the earth, and connected to the $50 \Omega$ input of a memory digital oscilloscope (Lecroy 9450, bandwidth $350 \mathrm{MHz}$ ). The oscilloscope input is protected by two fast diodes (AN4148, $100 \mathrm{~mA}, 4 \mathrm{~ns}, 100 \mathrm{~V}$ ) placed in head to head.

The trigger level of the oscilloscope is chosen the lowest possible in order to record the lowest energy streamers 
while avoiding inopportune triggering of the oscilloscope owed to partial discharge currents through the external walls of the test cell. This level is as more elevated than the electrode gap and the applied voltage is increased [2].

\section{Current shapes}

According to the amplitude of the applied voltage, different shapes of current can be observed [9]. They appear under the two alternations (positive and negative) when the voltage overtakes a certain threshold value corresponding to the streamers initiation. The positive ones apparition is however more frequent than the negative ones. Figure 1 shows the different shapes of current, observed in mineral oil under different voltages for the same geometry of electrodes.

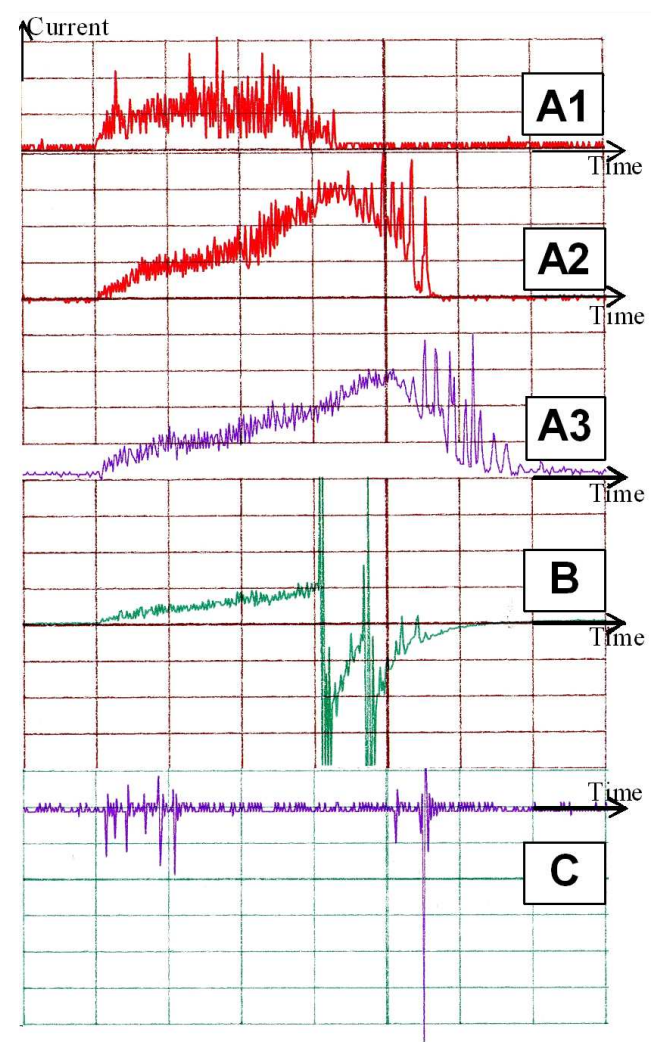

Fig. 1. Different types of recorded currents for applied voltage $U=27 \mathrm{kV}$, gap $d=12.5 \mathrm{~mm}$, point radius curvature $r_{\mathrm{p}}=10 \mu \mathrm{m}$. A $1, \mathrm{~A} 2$ and A3: positive currents not followed by arc discharges. B: positive followed by an arc discharge. C: negatives not followed by arc discharge. Scale: time: $2 \mu \mathrm{s} /$ div; currents: A1, A2, and A3: $5 \mathrm{~mA} /$ div; B: $10 \mathrm{~mA} /$ div and $\mathrm{C}: 1.5 \mathrm{~mA} /$ div.

Currents of type A1 are mainly recorded at the lowest voltage levels. They correspond to positive streamers propagation extinguishing before reaching the opposite electrode. Those of type A2 and A3 are the most frequently recorded at greater voltage levels. They are characterized by a continuous component that increases with time until a maximal value corresponding to the streamer arrival on the plane electrode $[10,11]$. Current pulses whose amplitude decreases with the applied voltage are superimposed on this continuous component. It is the type of current the most recorded [2].

Signals of type B are associated to positive streamers propagation followed by a breakdown of the whole insulation system. The breakdown can occur before or after reaching the maximal value of the current corresponding to the arrival of the streamer on the opposite electrode. This type of current is the most dangerous and should correspond to very fast events such as those observed by Torshin [12] and Lundgaard et al. [13]. The protection devices must identify them and grant them a particular interest.

Currents of type $\mathrm{C}$, recorded at further more elevated voltage levels than the previous ones, correspond to negative streamers propagation, which are slower and of weaker energy than the positive ones, and that extinguish frequently before reaching the opposite electrode. This type of streamer does not represent a significant immediate danger for the insulation system.

\section{Signals characterization}

The characterization of these types of signals is complex and difficult to perform. It requires the use of predictive models based on statistics and probabilities. The use of neural networks is better indicated for solving this type of problem which consists on electrical signals classification.

\subsection{Choice of the ANN architecture}

The choice of an ANN adequate architecture is primordial in order to obtain an effective system or at least functional.

Usually, only one hidden layer is sufficient to solve most problems. In general, more an ANN has inputs and more the solving of the problem is complex, more it will be necessary to add neurons in the hidden layer [14].

Moreover, concerning the choice between a forward and back propagation topology, and interconnections between neurons, the problem to solve has to be well known. For instance, a recursive structure is often better adapted for the recognition and classification of signals or pictures. It is also very effective when the temporal aspect of the applied signals in the input is important.

However, the topology of forward propagation networks remains the most used because of the simplicity of the used training algorithms and for its reputation to produce performing solutions to several concrete problems of the industry.

When the ANN must provide a response in real time, or furthermore, when it must be adapted to changes of its environment while pursuing its training in real time, the structure of the network must be then simplified at the maximum in order to decrease the number of required mathematical operations and to limit the necessary material resources to the implementation of the ANN. 


\section{Methodology}

\subsection{Neural network inputs}

In the present work, the neural model inputs will be determined from results obtained by the robust multi-linear regression line. For better results, input parameters must be most representative of the signals to identify.

\subsection{Input vector creation}

The digital signal (well sampled signal) representing the input vector passes by the following processing units:

Indentation of the signal: This unit enables to divide the signal of length $X$ in windows (segments) of data. One uses this technique to be able to introduce the signal segments one after the other by following the chronological order (simulation in real time). The Matlab command used in this unit is: buffer (signal of length $X$, size of the window). In order to avoid an excessive or slow indentation of the signal, only the critical zone corresponding to the rising zone of current is considered. All signals are divided into windows of equal size which are in their turn divided into sub-windows (Fig. 2). The Matlab command used in this unit is: buffer (window, size window $/ 5$ ).

Regression line: The regression line is represented by the equation: $y=A+B x$. It is defined by the parameters $A$ and $B$ that are determined by applying the least squares method

$$
\begin{aligned}
& A=\operatorname{cov}(x, y) / V(x), \\
& A=\left(\frac{\sum x y}{n}-\bar{x} \bar{y}\right) /\left(\frac{\sum x^{2}}{n}-\bar{x}^{2}\right), \\
& B=\bar{y}-A \bar{x},
\end{aligned}
$$

where $n$ is the number of observations.

Indeed, it consists in finding values of these two parameters that minimize the sum of the square of the deviations between the different points and the line itself.

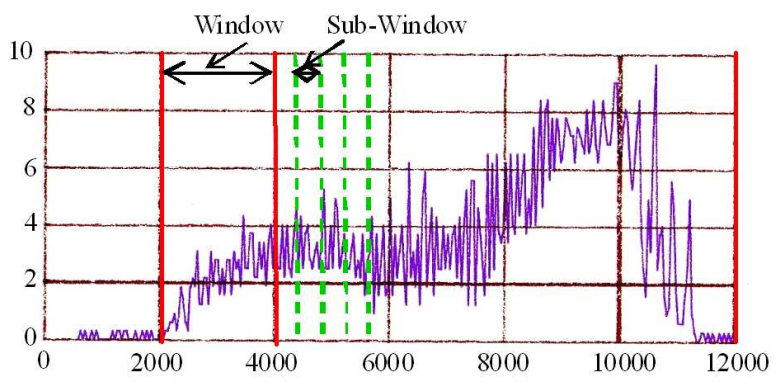

Fig. 2. Signal type and windows choice.

In this calculation unit, we will determine the equation ( $y=A+B x$ ) of the robust multi-linear regression line of a window, where $B$ represents the slope. This parameter represents the neural network principal input.

The advantage of the multilinear regression line is that it passes by the maximum of points with regard to the linear regression line. The Matlab command used in this unit is: robustfit (size of the window, window).

\subsection{Neural network output}

The output will be under binary form ( 1 or 0 ) for every window. A great neurons number increases the time of calculation exaggeratedly, but yields better results generally. Currently, there does not exist any method to find the optimal configuration. Several networks have been therefore tried and the better is kept. The retained configuration is a multilayer network with two hidden layers.

\section{Testing procedure}

The database contains signals of different current categories. A first ordering will be done at the creation of this database. Every signal portion will be classified according to its type (dangerous or not dangerous).

The database thus constituted will serve for the training phase of the neural network. Different types of training will be used, and the retained training will be the"trainlm". The best configuration of the ANN will be chosen according to its performances, from the table issued from the training phase.

The following phase consists in introducing discharge signals as inputs and to observe the behavior of the network.

\section{Testing and results}

Figures 3 and 4 are obtained from the test of the network. The blue color represents the original signal, and the red color - the result of the test. The latter evolves with the original signal until the network identifies it as being a dangerous signal. Then the circuit breaking can be triggered. Otherwise, it continues its evolution without any action order.

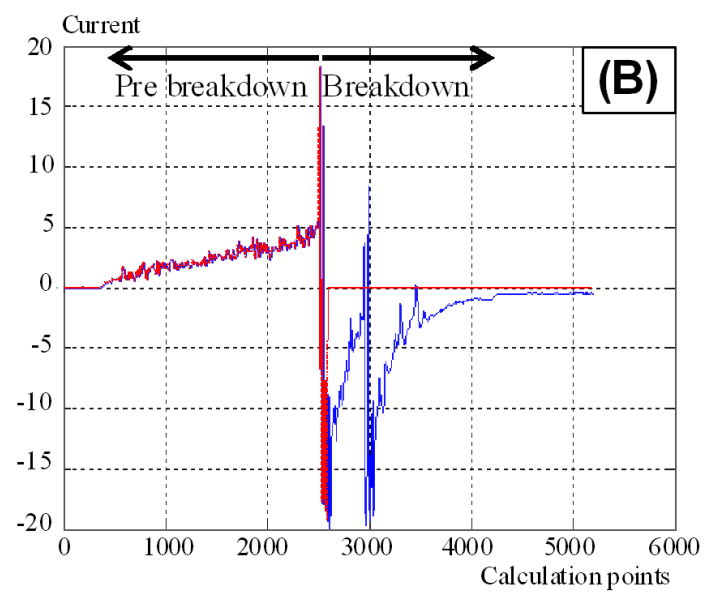

Fig. 3. Results of the test: current signal identified as leading to breakdown and instructions of breaking before arcing phase arriving. Scale: current: $2.5 \mathrm{~mA} / \mathrm{div}$, time: 300 calculation points $/ \mu \mathrm{s}$.

The two signals are superimposed until the computed signal (red color) will be set to zero. This means that a 


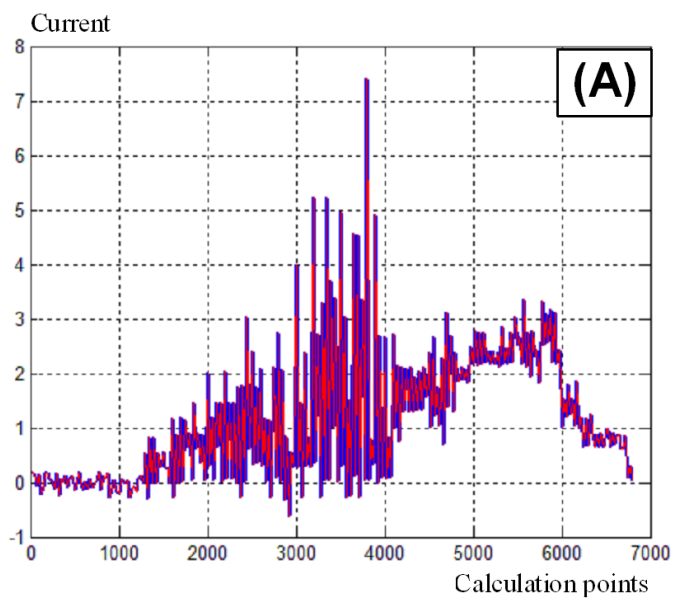

Fig. 4. Results of the test: recorded current signal and stored without breaking instruction as a function of the number of calculation points. Scale: current: $2.5 \mathrm{~mA} /$ div, time: 300 calculation points $/ \mu \mathrm{s}$.

dangerous window is detected and can be succeeded by an arc discharge event. This result is conclusive since the zero setting is instantaneously executed before the high energy arcing occurs. This can enable to prevent eventual equipment deterioration and circuit breaker acting with relatively high initial currents that can provoke a failure of breaking.

The red color signal (Fig. 4) superimposes perfectly with the signal of origin (blue color), which means that this signal is not immediately dangerous and will be classified as of second level. This type of signal does not present any potential risk to be followed by an arc discharge.

\section{Conclusion}

Power transformer oil current discharges present different characteristics as a function of the energy and the cumulated number of the discharges. They consist mainly in their continuous component shape, their amplitude, and the frequencies of the pulses that are superimposed to them. They depend on the physico-chemical properties of the oil related to their molecular structure and other new molecules and dissolved gases generated by the discharges.
Real time analysis of pre-discharge signals (default currents or corresponding emitted lights) represents a fast and reliable solution for transformers diagnosis monitoring and protection. The default current discrimination approach based on artificial neural networks coupled with regression line slope analysis proved out to be a real time and robust solution for solving this type of maintenance problem which depends on several parameters, and satisfactory results have been obtained.

\section{References}

[1] A. Beroual, M. Zahn, A. Badent, K. Kist, A.J. Schwabe, H. Yamashita, K. Yamazawa, M. Danikas, W.D. Chadband, Y. Torshin, IEEE Electr. Insul. Mag. 14, 6 (1998)

[2] A. Beroual, H. Moulai, Arch. Electr. Eng. (AEE) L, 115 (2001).

[3] V.G. Arakelian, IEEE Electr. Insul. Mag. 18, 26 (2002).

[4] M. Wang, IEEE Trans. Power Deliv. 18, 163 (2003).

[5] A. Sarikhani, E. Reihani, N. Nabizadeh, A. Hooshmand, M. Davodi, Europ. Trans. Electr. Power 19, 1140 (2008)

[6] IEEE Std. C57.104, Guide for interpretation of gases generated in oil-immersed transformer 2008.

[7] M. Duval, J. Dukarm, IEEE Electr. Insul. Mag. 21, $21(2005)$

[8] M. Duval, Recent Developments in DGA Interpretation, CIGRE Joint Task Force, Brochure D1.01/A2.11 (2006).

[9] H. Moulai, A. Nacer, A. Beroual, IEEE Trans. Diel. Electr. Insul. 19, 498 (2012)

[10] A. Beroual, R. Tobazeon, IEEE Trans. Electr. Insul. EI-21, 613 (1986)

[11] Y. Nakao, H. Itoh, S. Hoshino, Y. Sakai, H. Tagashira, IEEE Trans. Dielectr. Electr. Insul. 1, 383 (1994)

[12] Yu.V. Torshin, IEEE Trans. Dielectr. Electr. Insul. 2, 167 (1995)

[13] L. Lundgaard, D. Linhjel, G. Berg, S. Sigmond, in: 12th Int. Conf. on Conduction and Breakdown in Dielectric Liquids, ICDL'96, Roma (Italy), Rome 1996, p. 175.

[14] C.T. Lin, C.S.G. Lee, in: Neural Fuzzy Systems, Prentice-Hall, Englewood Cliffs 1996, p. 205. 